\title{
Questes
}

\section{Si fu ja fiex d'un nostre sers... Une remise en cause des textes autoritaires sur Pharamond}

\section{Sophie Albert}

\section{(2) OpenEdition}

12 Journals

\section{Édition électronique}

URL : http://journals.openedition.org/questes/1561

DOI : 10.4000/questes. 1561

ISSN : 2109-9472

\section{Éditeur}

Les Amis de Questes

\section{Édition imprimée}

Date de publication : 15 janvier 2006

Pagination : 21-29

ISSN : 2102-7188

\section{Référence électronique}

Sophie Albert, « Si fu ja fiex d'un nostre sers... Une remise en cause des textes autoritaires sur

Pharamond », Questes [En ligne], 8| 2006, mis en ligne le 01 janvier 2014, consulté le 07 mai 2019.

URL : http://journals.openedition.org/questes/1561 ; DOI : 10.4000/questes.1561

Ce document a été généré automatiquement le 7 mai 2019.

(c) Association des amis de «Questes » 


\title{
Si fu ja fiex d'un nostre sers... Une remise en cause des textes autoritaires sur Pharamond
}

\author{
Sophie Albert
}

1 Au milieu de la dernière séquence du roman de Guiron le Courtois ${ }^{1}$, consacrée aux amours malheureuses de Guiron et de Bloie ${ }^{2}$, l'auteur s'arrête, en vertu du principe de l'entrelacement, sur les aventures de Brehus sans Pitié ; il relate ses démêlés avec une demoiselle dont il est passionnément épris, mais qui n'aspire qu'à le tuer et qui, dans ce dessein, le précipite dans une caverne magnifiquement décorée et sculptée. En cherchant à sortir de la grotte, Brehus entre dans une première chambre, où il voit un homme mort, étendu sur un lit; dans sa main, un « brief » révèle qu'il a pour nom Febus. Après avoir lu le parchemin, Brehus traverse trois autres chambres, toutes occupées par des tombeaux. Alors qu'il vient de faire ses prières dans une dernière chambre où se tient un autel, un vieux chevalier entre. Les deux hommes en viennent à parler de Guiron, dont Febus est le bisaïeul et le vieillard, le grand-père paternel. Il renseigne Brehus sur le lignage du héros : Le roiaume de Gaulle deüst estre nostres par raison, car li sire qui laiens gist si en eüst esté rois, s'il vauxist : mais il ne vaut la segnourie, ains la douna un soen frere mainsné de lui. Il fu chevalier de grant cuer et de si haute afaire qu'il ne vaut la couroune recevoir : et ele devoit estre soe, car il estoit li ainsnés freres ; il laissa la couroune pour entendre a chevalerie.

Li rois Clodeus, qui fu li premiers rois crestiens de Gaulle, fu commencement de no lignage, de ceus qui chrestiens furent. Encor vous di ge une autre chose : or saciés que li roialmes de Gaulle devroit estre de Guron, car, avant que li rois Faramons en fust corounés, em porta la couroune li peres de Guron ; et puis por dolour de moi se mist il ceiens en ceste habitation que vous ceens veés et douna celui roialme a un suen neveu. Le rois Faramont, qui orendroit porte la couroune de Gaulle, si fu ja fiex d'un nostre sers, que ge commandai a franchir. Quant cil morut a cui mes fiels avoit douné le roialme de Gaulle, il morut sans oir : Faramont, qui assés savoit mal et bien, entra adonc dedens la terre : ensint com ge sai, il i entra desloialment. 
Guron ne set rien de tout ceu, il ne set de cui il fu fiex, il cuide bien que ses peres soit mors piecha : et pour ce ne sauroit il ore que respondre de toute ceste chose, qui l'en vaudroit demander la verité. Guron, il est moult gentill home : il fu estrais de par sa mere del lygnage Helayn le Gros, qui fu neveu Josept de Barimatie [... $]^{3}$.

2 Comme le comprend le lecteur, Guiron devrait, selon son sang, régner sur le royaume de Gaule. Or il n'en est rien, et l'explication que propose l'auteur a de quoi surprendre : il imagine de faire de Pharamond un fils de serf, un usurpateur qui, bafouant les droits de la lignée royale, se serait emparé «desloialment» du pouvoir. Ce faisant, il s'inscrit en porte-à-faux vis-à-vis de plusieurs textes "autoritaires ", les généalogies de souverains français et le Tristan en prose, dont je vais dans un premier temps indiquer la teneur.

\section{Fortunes de Pharamond}

3 À l'arrière-plan de la littérature, Pharamond figure, à partir du Liber Historiae Francorum (727), dans la plupart des généalogies des rois de France, qui font de lui un rejeton de la race troyenne $e^{4}$. Maillon de la lignée royale, père des rois mérovingiens, ce personnage, inventé de toutes pièces au début du viII ${ }^{\mathrm{e}}$ siècle, s'inscrit dans la longue élaboration d'une histoire nationale française. Les Grandes Chroniques de France (1274) le placent à l'origine de la lignée mérovingienne, consacrant sa légitimité ${ }^{5}$. Plus tard, le $\mathrm{xv}^{\mathrm{e}}$ siècle mettra en place une différenciation entre Clovis et Pharamond - Clovis sera le roi très-chrétien, Pharamond un législateur, fondateur de la loi salique ; la faveur du Mérovingien atteindra son apogée pendant les Temps Modernes. Ainsi, si le personnage ne jouissait pas au XIII ${ }^{\mathrm{e}}$ siècle du crédit qui sera le sien à partir $\mathrm{du} \mathrm{xv}^{\mathrm{e}}$, il faisait déjà partie de plein droit de la généalogie des souverains français ${ }^{6}$.

D'autre part, Pharamond entre dans la littérature française au début du Tristan en prose, roman arthurien dont les premières rédactions datent des années $1230-1235^{7}$. Ce début se présente à bien des égards comme la transposition, sur le mode de la fiction, d'une chronique "européenne», qui commence avec l'arrivée en Occident de Joseph d'Arimathie. Le lecteur découvre une Grande-Bretagne et une Gaule païennes, lentement christianisées. Telle est la première mention de la Gaule :

50. A celi tens que je vos cont estoit li reaumes de Cornoaille et celi de Leonois en la subjection au roi de Gaule. Cornoaille rendoit au roi de Gaule cent demoiseles et cent demoisiax et cent chevax de pris ; autresi faisoit Lionois. Et tenoit li rois de Gaule de la seignorie de roïne. Et sachiez que a celi tens rendoient totes les regions dou monde rentes et treü a roïne. Ne n'avoit encores a celi tens nul crestien en Gaule, enz erent tuit paien, et avoient divers diex et diverses creances. Et li rois qui adonc regnoit en France estoit apelez Marovex, bons chevaliers durement, et preudons de sa loi. Et sachiez que aprés sa mort ne demora pas granment a venir Saint Remi a la loi crestiene, qui converti Cledovex, qui fu li premiers rois crestiens de France ${ }^{8}$.

5 L'auteur précise le régime politique du royaume de Gaule et des terres environnantes, le tribut, souvent lié, dans les romans en prose, au paganisme ; en anthropologue attentif, il fournit au lecteur une définition de la loi païenne, qui amène à vénérer « divers diex et diverses creances ». La généalogie des rois, seulement ébauchée dans ce premier extrait, s'étoffe ensuite. 
140. [...] li bons rois Maronex, sires de Gaule, avoit esté mors tot novelement. Childeris, ses filz, reignoit, qui assés estoit mauvais rois e coars, e maintenoit mauvaisement sa terre, que ses peres avoit tenue en pais?

205. Cele semene meïsmes que la chose fu ensi avenue, vindrent unes letres au roi Apollo, que li rois Clodoveux li mandoit qu'il fust a son coronement en cel mois, et amenast avec soi la roïne, sa feme, et ces qu'il voudroit de sa terre. Et cil Clodoveux fu novelement tornez a la loi crestiene, et grant partie de sa gent ; et grant partie en $i$ avoit qui ne voloient la loi des paiens lessier. Et sachiez que Saint Remi l'avoit converti a la loi crestiene ; et encores demoroit il en France, et aloit preschant aus mescreanz le non dou vrai crucefi ${ }^{10}$.

6 Clovis, comme dans la première mention qui lui est consacrée, est étroitement associé à la légende de saint Rémi et, donc, au christianisme : c'est bien le passage d'une « loi » à une autre qui est en jeu.

7 Mérovée, Childéric et Clovis sont supposés vivre plusieurs générations avant les temps arthuriens et la naissance de Tristan. Pharamond intervient plus loin dans le roman, alors que Tristan, tout jeune enfant, cherche un asile à la cour de France ${ }^{11}$. Pharamond, dans les chroniques, est l'aïeul de Clovis, et non son descendant. Tristan et, à sa suite, Guiron inversent l'ordre: Clovis devient un ancêtre, Pharamond un contemporain. Le légendaire roi de Gaule, rajeuni d'une bonne centaine d'années, perd son statut d'ancêtre. De cette distorsion chronologique, le lecteur ne doit pas s'étonner: on trouve de nombreux exemples similaires dans les chroniques elles-mêmes et dans les textes littéraires. Comme l'écrit Richard Trachsler, « ce télescopage (entendons : superposition de deux époques au prix d'une légère entorse à la chronologie 'historique') ne posait pas de problème $»^{12}$. Il devait être tentant de joindre en un même roman les figures emblématiques de deux histoires nationales - la France mérovingienne de Pharamond, la Bretagne d'Arthur.

Ce bouleversement chronologique a pour effet de «christianiser» Pharamond. Les chroniques précisent fréquemment que ce roi est païen - et pour cause : il faudra attendre Clovis pour que la royauté française se convertisse à la « vraie foi ». Dans Tristan en revanche, Pharamond arrive sur le trône en un temps où les rois sont chrétiens depuis belle lurette. Baptisé, sacré sans aucun doute, le personnage sort grandi de l'infléchissement que Tristan impose aux chroniques. Au demeurant, tout dans le roman contribue à donner de lui une image positive. Son « ostel » est présenté comme un lieu où l'on peut, mieux qu'ailleurs, accroître ses qualités chevaleresques: comme le dit Gouvernal à Tristan, «la aprendrez vos a servir et a cortoier, et coment hauz hom et gentils hom se doit maintenir $»^{13}$. Pharamond, loin de décevoir les attentes du précepteur, reçoit «mout volentiers » Tristan et Gouvernal ; quelques années plus tard, il manifeste un sens aigu de la justice en tranchant un litige qui oppose Tristan à sa propre fille. Un roi juste et courtois, un asile hospitalier pour Tristan : le tableau, pour être bref, n'en est pas moins celui d'une cour brillante, gouvernée par un souverain exemplaire.

9 Guiron le Courtois, en attribuant à Pharamond une origine servile, remet ainsi en cause deux corpus autoritaires : dans le champ historiographique, les chroniques latines ayant inventé, puis véhiculé le nom du premier roi de France; dans le champ littéraire, le Tristan en prose, principal intertexte du roman, dont l'intrigue, plusieurs fois mentionnée sous forme de prolepses, est supposée faire suite à celle de Guiron. Le «noircissement » de Pharamond déroge au procédé de la rétrodiction ${ }^{14}$, qui impose théoriquement de respecter les données des textes antérieurs. Je vais maintenant proposer quelques éléments d'explication à ce déni d'autorité. 


\section{Pourquoi l'origine servile de Pharamond?}

10 source dans l'hostilité de l'Italie, où le passage est copié dès la fin du XIII ${ }^{\mathrm{e}}$ siècle, envers la royauté française ${ }^{15}$. L'hypothèse se heurte à une première objection: les plus anciens manuscrits connus, probablement antérieurs aux traductions italiennes, dénoncent déjà la naissance servile et la déloyauté de Pharamond ${ }^{16}$. Quant aux manuscrits français des $\mathrm{XIV}^{\mathrm{e}}$ et $\mathrm{XV}^{\mathrm{e}}$ siècles, ils conservent la leçon, comme s'en étonne le critique : «ce trait, pour le moins inamical à l'égard de la France, n'a pas empêché la diffusion du texte dans le pays gouverné par les Capétiens directs, puis par les Capétiens-Valois $»^{17}$. Un autre élément invite à reconsidérer l'hypothèse de Venceslas Bubenicek. La mention de Clovis, qui apparaît en promoteur du christianisme dans la généalogie de Guiron, contribue à magnifier son lignage plutôt qu'à l'assombrir. On voit mal pourquoi l'auteur aurait voulu, d'un côté, calomnier les souverains français en frappant d'opprobre le personnage de Pharamond et, de l'autre, présenter Clovis sous un jour positif.

La stigmatisation de Pharamond s'explique peut-être par des raisons purement littéraires, qui tiennent d'abord à la logique propre du roman. Guiron le Courtois devait concilier deux exigences contradictoires. Tristan faisait entrer Pharamond dans l'histoire romanesque de la Gaule ; Guiron, « suite rétrospective » de Tristan, ne pouvait manquer de placer à son tour Pharamond sur le trône. Mais il lui fallait également justifier que Guiron ne puisse pas devenir roi de Gaule, alors même que son ascendance l'y autorisait. « Noircir » Pharamond était pour l'écrivain un moyen efficace d'articuler les données des textes autoritaires avec sa propre version des faits. C'était, surtout, une solution plausible. Pharamond, dans les chroniques, n'existait guère que par son nom. On pouvait le doter d'attributs fantaisistes sans pour autant remettre en cause les textes historiographiques, très flous à son sujet - lui attribuer par exemple une origine servile plus facilement qu'à Clovis, dont la légende, au milieu du XIII ${ }^{\mathrm{e}}$ siècle, était bien constituée ${ }^{18}$.

12 La fiction introduite par l'auteur de Guiron n'est pas sans évoquer des thèmes familiers aux lecteurs médiévaux, qui touchent à la représentation du pouvoir et de la royauté. Les histoires d'imposture abondent depuis le début du XII ${ }^{\mathrm{e}}$ siècle. La réalité fournissait là aux écrivains, à défaut de modèles, des exemples nombreux. Aussi, comme beaucoup d'imposteurs réels, Pharamond se glisse dans un «trou » de la succession lignagère : le neveu du précédent roi meurt "sans oir ", laissant une place vacante ${ }^{19}$. Ces histoires d'imposture, d'autre part, donnaient lieu à plusieurs discours, discours autorisé du pouvoir légitime ou discours sulfureux des transfuges. L'imposteur, invariablement, était accusé par ses détracteurs d'être issu «de basse geniture » et d'agir contre la justice. Résumé à l'extrême dans le portrait de Pharamond, ce discours n'y apparaît pas moins en filigrane ${ }^{20}$.

Parallèlement, dans la littérature arthurienne, le motif est fréquent de la succession royale entravée : que l'on pense simplement au Merlin, à la difficile accession au pouvoir d'Uter et Pandragon, ou à la manière dont Arthur voit discutés ses droits. L'antagonisme entre Guiron et Pharamond rappelle notamment les rapports d'autres héros à des rois malveillants. Au début du Lancelot en prose, le roi Claudas envahit les terres du roi Ban, privant Lancelot de son domaine et, par là, de la royauté. Claudas, dans Guiron, est un allié 
indéfectible du roi Pharamond ${ }^{21}$. N'est-ce pas signifier l'alliance de deux souverains néfastes qui pervertissent, chacun à sa manière, les lois de succession? Dans chaque cas, le héros éponyme, dépouillé de la royauté par un personnage sans scrupule, est projeté dans l'univers des chevaliers errants.

Ainsi, pour justifier simultanément la royauté avortée de Guiron et l'usurpation de Pharamond, l'auteur s'appuie sur l'entrecroisement de deux types de discours. Premier discours, appliqué à Pharamond, celui des pourfendeurs de l'imposture, qui taxe l'imposteur d'une double bassesse, morale et sociale. Second discours, celui du parcours héroïque, qui met en scène un héros temporairement caché ou méconnu, puis reconnu et rétabli dans ses droits ${ }^{22}$. Guiron, dont tous les chevaliers errants, à commencer par lui, ignorent l'ascendance, correspond en partie au modèle. Mais la reconnaissance n'a pas lieu : l'identité du héros demeure pour jamais dans l'ombre, le schéma est incomplet.

Le fait que Guiron ne soit pas reconnu rejaillit sur le sens de l'accusation portée contre Pharamond et, surtout, sur la nature exacte de son rapport avec les textes autoritaires. Comme l'origine royale de Guiron, l'origine servile de Pharamond est marquée par le sceau du secret. En effet, après avoir raconté à Brehus l'histoire familiale de Guiron le Courtois, l'aïeul lui fait promettre de ne rien révéler de ce qu'il a appris :

Vous m'avés otroié que a home ni a feme vous ne dirois nouveles de nous ne de chose que vous aiés veü chaiens, ensint que pour acoison de vos paroles viegne chaiens home ne feme ${ }^{23}$.

La révélation du lignage, confinée dans l'espace de la caverne, «n'informe ni ne relance le récit $\aleph^{24}$; Guiron, ne sachant rien, ne pourra être tenté de reprendre la Gaule à son illégitime souverain. Le montage littéraire que met en place l'auteur de Guiron le Courtois lui permet d'opposer un contredit aux représentations traditionnelles de Pharamond tout en empêchant que ce contredit déborde les limites du roman, voire de l'épisode: l'autorité est écornée, mais elle est sauve.

\section{NOTES}

1. Pour lire un résumé de ce roman, daté des années 1235-40, voir LATHUILLÈRE, Roger, «Guiron le courtois " : étude de la tradition manuscrite et analyse critique, Genève, Droz, 1966. Les numéros de paragraphes renvoient à cet ouvrage, les numéros de folios au ms. de Paris, BNF f. fr. 350.

2. $\$ 91-130, f^{\circ} 239-354$.

3. $\S 111, \mathrm{f}^{\circ} 289 \mathrm{~d}$; LIMENTANI, Alberto, Dal « Roman de Palamedés » ai «Cantari di Febus-el-Forte » : testi francesi e italiani del Due e Trecento, Bologna, Casa Carducci, 1962, pp. 74-76.

4. Liber Historiae Francorum, ou Gesta Regum Francorum, dans MGH, Scriptorum rerum Merovingicarum , KRUSCH, Bruno (éd.), Hanovre, t. II, 1984 (première édition : 1888), pp. 215-329. Voir aussi le Recueil des historiens des Gaules et de la France, éd. Dom Martin Bouquet, nouvelle édition : Paris, Victor Palmé, t. II, 1869-1880, qui a le mérite de présenter une quantité considérable de généalogies.

5. Les Grandes Chroniques de France, VIARD, Jules (éd.), Paris, Société de l'Histoire de France, t. I, 1920, pp. 19-20. 
6. Sur la place de Pharamond dans la construction légendaire de la royauté française, voir BEAUNE, Colette, Naissance de la nation France, Paris, Gallimard, 1985.

7. Le Roman de Tristan en prose, CURTIS, Renée Lilian (éd.), München, M. Hueber, t. I, 1963. Les citations qui suivent sont extraites de cette édition. Un dénommé Aramon apparaît dans le Lancelot en prose, mais son identification à Pharamond demeure éminemment douteuse.

8. Ibid., p. 58.

9. Ibid., p. 94.

10. Ibid., p. 118.

11. Ibid., §261-281, pp.137-145 pour le passage où Pharamond apparaît. Tristan reste chez Pharamond entre sa huitième et sa douzième année.

12. TRACHSLER, Richard, Disjointures, Conjointures. Étude sur l'interférence des matières narratives dans la littérature française du Moyen Âge, Tübingen, A. Francke Verlag, 2000, p. 238 ; voir aussi la p. 253.

13. Le Roman de Tristan en prose, éd. cit., p. 137.

14. Terme employé par Richard Trachsler à propos du Roman de Perceforest, "suite rétrospective " du Lancelot-Graal, et défini comme un procédé « où il s'agit d'expliquer, de préférence de manière originale, des faits relatés ailleurs en 'extrapolant' à partir des textes déjà en place » ( Disjointures..., op. cit., pp. 241-242).

15. BUBENICEK, Venceslas, «Quelques figures de rois chevaliers errants dans le roman en prose de Guiron le Courtois », Bien dire et bien aprandre, 17 (1999), pp. 49-61.

16. Voir notamment le ms. de Marseille, BM 1106, daté des années 1260-1270, fo 170d (même leçon, au mot près, que le ms. 350).

17. BUBENICEK, Venceslas, "Quelques figures... », art. cit., p. 58.

18. De ce point de vue, on peut même se demander, vu les distorsions que le Tristan en prose, puis Guiron font subir aux chroniques, si celles-ci ont vraiment valeur d'autorité au XIII ${ }^{\mathrm{e}}$ siècle. Peutêtre faut-il attendre la traduction française, fixation de l'histoire nationale, pour que cette histoire soit perçue comme une autorité.

19. Voir LECUPPRE, Gilles, L'imposture politique au Moyen Âge : la seconde vie des rois, Paris, PUF, 2005, notamment pp. 256-261.

20. Ibid., pp. 50-53.

21. Voir les $\S 38$ et 49-50 de l'Analyse. Dans le Tristan en prose, Pharamond est l'ami du Morholt, trait repris dans Guiron; or le Morholt, tout valeureux qu'il soit, n'en est pas moins le percepteur du tribut dont s'acquitte la Cornouaille.

22. Exemple typique, Arthur dans le Merlin en prose, élevé par Auctor, vassal du roi, qui est reconnu au moment de son adolescence, par l'élection divine puis par la parole merlinienne.

23. LimentAni, Alberto, Dal « Roman de Palamedés »..., éd. cit., p. 187. En réalité, Brehus a le droit de dire la vérité à Guiron; mais dans la version de base, il n'aura jamais l'occasion de raconter au héros son histoire. Voir WAHLEN, Barbara, «Du recueil à la compilation : le manuscrit de Guiron le Courtois, Paris, BNF fr. 358-363 ", Ateliers, 30 (2003), pp. 89-100.

24. WAHLEN, Barbara, « Du recueil à la compilation », art. cit., p. 95.

\section{INDEX}

Mots-clés : autorité, Pharamond, amour, héros, pouvoir, Fortune, servilité

Keywords : authority, Pharamond, love, hero, power, Fortune 